\title{
Prevalencia de leptospirosis en adultos Chile, 2003
}

\author{
Solana Terrazas, Andrea Olea, Stella Riedemann y Marisa Torres
}

\section{Prevalence of leptospirosis in adults in Chile, 2003}

Background: Leptospirosis is a zoonosis endemic in some regions in Chile. Since its inclusion into the list of notifiable diseases, the Chilean Ministry of Health was able to maintain an adequate surveillance of leptospirosis. Nonetheless, some cases are not reported due to subclinical disease or nonspecific symptoms. Objectives: Determine the national prevalence of leptospirosis and assess the epidemiological characteristics of seropositive individuals. Methods: Secondary data analysis of the National Health Survey, 2003. Results: National prevalence was $0.4 \%$. Low socioeconomical status and female gender were characteristics, which were more frequently found in seropositive cases. The most common serovars were icterohaemorrhagiae, bratislava and pomona. Conclusion: We present the first epidemiological analysis of leptospirosis on a national level in Chile. Thus, the study contributes to the knowledge the epidemiological situation of this disease in Chile.

Key words: Leptospirosis, prevalence, risk factors

Palabras clave: Leptospirosis, prevalencia, factores de riesgo

\section{Introducción}

$\mathrm{L}$ a leptospirosis es una zoonosis de distribución mundial, endémica en zonas con clima tropical y en algunos países de clima templado, como ciertas regiones de Chile. Su incidencia en humanos varía dependiendo de la zona geográfica, con tasas entre 0,1-1/100.000 habitantes en países con clima templado y $10-100 / 100.000$ habitantes en países tropicales ${ }^{1}$. La prevalencia en población general también varía dependiendo del área geográfica que se estudie, pudiendo estar en alrededor de 1,2 a $6 \%$ en estudios recientes en poblaciones de América del Sur ${ }^{2,3}$. Por otro lado, se han realizado estudios de prevalencia los últimos 20 años, en grupos de población ${ }^{4-7}$, grupos de alto riesgo ${ }^{8-15}$ y contactos de enfermos ${ }^{16}$, encontrándose resultados variables dependiendo de la zona y la ocupación, pero donde los factores de riesgo se repiten: exposición a aguas contaminadas, manipulación de animales, contacto con animales, regiones con inundaciones frecuentes y áreas pobres y rurales. Sin embargo, se estima que su magnitud está subestimada debido a su amplio espectro clínico y a la falta de métodos diagnósticos que sean sensibles y específicos ${ }^{17}$. Se ha demostrado la alta tasa de sub-notificación existente debido a que muchos casos de enfermedad tienen una presentación subclínica. Estudios realizados en Brasil en pacientes con dengue y hepatitis viral han demostrado que entre 9 y $15 \%$ de estos pacientes presentan además positividad para anticuerpos recientes anti leptospira ${ }^{18}$, sugiriendo que la sub-notificación puede ser aún más alta cuando en los casos existen dos patologías similares concomitantes.

Leptospira spp tiene como reservorio algunas especies de mamíferos, principalmente los de pequeño tamaño. La leptospirosis puede ser desarrollada por el humano y por algunas especies de mamíferos que actúan como hospedero accidental. Los portadores no son hospederos accidentales, los serovares, en general, se han adaptado a ellos y viceversa. Los animales que pueden presentar la enfermedad son principalmente animales domésticos (caninos) y los reservorios son generalmente ratas, bovinos y porcinos. Los caninos también son reservorios de algunos serovares ${ }^{19,20}$.

Estudios en seres humanos han identificado algunos grupos de riesgo, principalmente relacionados con ocupación, como: veterinarios, limpiadores de alcantarillados, mineros, faenadores de animales y soldados, actividades recreacionales donde hay inmersión en el agua (por inmersión en aguas contaminadas), habitantes de zonas rurales y también en zonas urbanas, principalmente por contacto con caninos no vacunados ${ }^{21}$. Estudios realizados en Chile han identificado como factores de riesgo el desarrollar labores agropecuarias, tener contacto con productos de animales contaminados y con agua de acequias, limpieza de lugares donde se ha detectado la presencia de roedores y falta de protección personal en ocupaciones donde existe un alto riesgo de infección ${ }^{8,13-15}$.

La leptospirosis se incorporó como Enfermedad de Notificación Obligatoria (ENO) en Chile a partir del año 2002. Los últimos brotes nacionales que se han producido han sido consecuencia de exposición ocupacional ${ }^{8}$ y recreacional ${ }^{22}$.

Para el efecto de las $\mathrm{ENO}^{23,24}$, se define caso sospechoso como: fiebre de inicio brusco, cefalea, mialgia (principalmente pantorrillas y región lumbar) con alguno de los siguientes signos y/o síntomas:
Pontificia Universidad Católica de Chile.

Facultad de Medicina

Departamento de Salud Pública (ST, MT*).

Departamento de Epidemiología Ministerio de Salud (AO). Actualmente en Facultad de Medicina Clínica AlemanaUniversidad del Desarrollo, Santiago. Centro de Epidemiología y Políticas de Salud.

Universidad Santo Tomás, Valdivia, Chile (SR).

*Actualmente en Universidad Católica del Norte, La Serena, Chile.

Financiamiento: ninguno Conflictos de interés: ninguno.

Recibido: 21 de marzo de 2011 Aceptado: 15 de septiembre de 2012

Correspondencia a: Solana Terrazas M. solanat@med.puc.cl 
- Postración/malestar general, irritación conjuntival, irritación meníngea (rigidez de nuca), insuficiencia renal (anuria, oliguria y/o proteinuria), ictericia, manifestaciones hemorrágicas (intestinales y pulmonares), arritmia o insuficiencia cardíaca, disnea, otros (náuseas, vómitos, dolor abdominal, diarrea y artralgias).

Además de los síntomas debe presentar algún antecedente de exposición a orina de animales o a animales infectados, como sucede con los cazadores ya que ellos mismos procesan sus presas, agua o lodo que pudieran haber estado contaminados, entre 4 y 20 días previos al inicio de los síntomas. El caso confirmado es todo aquel cuya etiología sea documentada por laboratorio.

Todas las sospechas de casos deben ser notificadas. A los pacientes se les debe aislar e iniciar una investigación epidemiológica para identificar posibles nuevos casos entre sus contactos y la fuente de infección. El tratamiento es principalmente sintomático para casos leves y se advierte la necesidad de buscar ayuda profesional en caso de aparecer ictericia. El tratamiento antimicrobiano ha demostrado su utilidad en los casos más graves. Los esquemas terapéuticos que incluyen penicilina o doxiciclina podrían ser útiles en esta enfermedad; sin embargo, no hay evidencia suficiente para hacer una recomendación general o un protocolo de tratamiento ${ }^{23,24}$.

Desde que se incorporó esta enfermedad en la lista de las ENO, se ha logrado mantener una adecuada vigilancia e identificar brotes precozmente, a pesar de que sabemos que esta cifra está sub-estimada dada la gran cantidad de casos sub-clínicos y con manifestaciones inespecíficas que no consultan. El objetivo de este estudio es determinar la prevalencia nacional de infección por leptospiras, conocer algunas características clínico-epidemiológicas de individuos seropositivos para leptospirosis y explorar diferencias y similitudes entre los individuos y con los factores de riesgo clásicamente descritos en la literatura científica.

\section{Material y Método}

Estudio de prevalencia, descriptivo, de corte transversal.
Creación de bases de datos: Los datos analizados son secundarios, obtenidos a partir de bases de datos creadas por los organismos responsables de cada estudio. Se utilizaron tres bases de datos (Tabla 1):

Base de datos del Estudio de Prevalencia de Enfermedades Prioritarias 2003. Este estudio del Ministerio de Salud, se llevó a cabo entre abril y diciembre de 2003 con el objetivo de medir la prevalencia de 23 enfermedades en la población general ${ }^{25}$. Se tomó una muestra representativa de la población chilena mayor de 17 años teniendo como marco de muestra la Encuesta de Calidad de Vida y Salud realizada el año 2000. De los 6.228 individuos entrevistados el año 2000, se tomó una muestra de 3.619 personas siguiendo la estructura de edad, sexo y región geográfica y con un sistema de reemplazos que permitió cumplir con el número y distribución de la muestra calculada inicialmente. A los individuos seleccionados se les realizó una encuesta y un examen de salud para las 23 patologías seleccionadas. También se tomaron muestras biológicas a partir de las cuales se creó una Seroteca Nacional conservada en el Instituto de Salud Pública de Chile (ISPCH). La seroteca cuenta con 3.118 muestras alicuotadas e identificadas en los laboratorios regionales, conservadas a $-70^{\circ} \mathrm{C}$ desde su recolección ${ }^{25}$.

Base de datos de la Encuesta de Calidad de Vida y Salud 2000. Esta encuesta del Ministerio de Salud, fue realizada durante el año 2000 para conocer la percepción que tiene la población sobre su vida y su salud y evaluar el impacto de las políticas sanitarias relacionadas con estos tópicos ${ }^{26}$. Las áreas temáticas abordadas en esta versión de la encuesta fueron: calidad de vida, estilos de vida y entorno, daño crónico y discapacidad y daño agudo y accidentes. La muestra se tomó en base al marco del Programa Integrado de Encuesta de Hogares del INE y se constituyó por 6.228 hogares con cobertura nacional, regional y urbano-rural.

Base de datos del análisis de las muestras de suero. Esta base de datos fue creada a partir del análisis de las muestras obtenidas de la Seroteca Nacional, creada a su vez, a partir del Estudio de Prevalencia de Enfermedades

\begin{tabular}{|c|c|c|c|c|}
\hline Estudio & Año & Objetivo del estudio & Muestra (n) & Organismo responsable \\
\hline $\begin{array}{l}\text { Estudio de prevalencia de enfermedades } \\
\text { prioritarias }\end{array}$ & 2003 & $\begin{array}{l}\text { Medir la prevalencia de } 23 \text { enfermedades prioritarias en la } \\
\text { población general }\end{array}$ & 3.619 & $\begin{array}{l}\text { Pontificia Universidad Católica de Chile } \\
\text { Ministerio de Salud }\end{array}$ \\
\hline Encuesta de calidad de vida y salud & 2000 & $\begin{array}{l}\text { Conocer la percepción que tiene la población sobre su vida y } \\
\text { su salud y evaluar el impacto de las políticas sanitarias relacio- } \\
\text { nadas con estos tópicos }\end{array}$ & 6.228 & $\begin{array}{l}\text { Ministerio de Salud } \\
\text { Ministerio de Planificación }\end{array}$ \\
\hline Análisis de las muestras de suero & 2007 & $\begin{array}{l}\text { Conocer la prevalencia nacional de leptospirosis a partir de } \\
\text { alícuotas guardadas en Seroteca Nacional. }\end{array}$ & 2.889 & $\begin{array}{l}\text { Proyecto Mideplan, Ministerio de Salud } \\
\text { Universidad Austral }\end{array}$ \\
\hline
\end{tabular}


Prioritarias 2003. De las 3.118 muestras guardadas en la seroteca del ISPCH, 2.861 fueron enviadas en tubos Eppendorf desde el ISPCH en Santiago hasta Valdivia, a la Universidad Austral, donde fueron analizadas. Las muestras se transportaron en cajas y cada tubo estaba rotulado con el número identificador y las iniciales de cada individuo. A partir del análisis de estas muestras se creó una base de datos con 2.889 individuos (28 muestras repetidas), donde se informa el número identificador del individuo o las iniciales rotuladas en los tubos y el resultado de la prueba aplicada. En el caso que el individuo resultara positivo, también se indicaba los títulos de anticuerpos y el serovar correspondiente.

Análisis de laboratorio: Las alícuotas se examinaron mediante la técnica de microaglutinación en tubo (MAT), prueba de elección en estudios de prevalencia, en la Universidad Austral de Valdivia. Se utilizaron como antígenos cultivos vivos de los serovares: hardjo, pomona, canicola, ballum, icterohaemorrhagiae, autumnalis, grippotyphosa, tarassovi y bratislava. Generalmente se recomienda usar títulos > a 100 para evidenciar exposiciones pasadas pero, dependiendo de la especie, se pueden usar títulos menores; por ejemplo, en humanos y roedores se comienza con la dilución 1:50 y si es positivo se titula hasta la dilución que se identifica como título final ${ }^{13,27}$. En primer lugar se realizó un tamizaje para descartar muestras negativas a los diferentes serovares. Los sueros que resultaron positivos fueron titulados en base a diluciones dobles del suero utilizando en ambos casos un control del $50 \%$ para cada uno de los serovares. Se consideró positivo títulos sobre 1:50.

Análisis de datos: Para el cálculo de la prevalencia nacional se parearon los resultados obtenidos en el laboratorio con la base de datos del Estudio de Prevalencia de Enfermedades Prioritarias 2003 a partir del número identificador y la fecha de nacimiento, logrando parear los resultados en 2.694 individuos.

Para la exploración de las características epidemiológicas de individuos seropositivos se creó una nueva base de datos donde se incluyeron todos los individuos que resultaran positivos para leptospirosis con MAT. Luego, se identificó cada individuo positivo en la base de datos del estudio de Prevalencia de Enfermedades Prioritarias 2003 y en la Encuesta de Calidad de Vida y Salud 2000 a partir de su número identificador. Se identificaron 6 casos positivos para leptospirosis de los cuales todos fueron encontrados en el estudio de Prevalencia de Enfermedades Prioritarias 2003 y 3 sólo en ambas bases de datos (2000 y 2003).

Se calculó la prevalencia de leptospirosis en la población general utilizando el factor de expansión determinado por el Estudio de Prevalencia de Enfermedades Prioritarias 2003 y luego se procedió a analizar los individuos por separado, para generar una descripción de cada uno de ellos. Posteriormente, se analizaron en conjunto para ver similitudes y diferencias entre ellos.

Para la caracterización de los casos que resultaron positivos al MAT, se incluyeron en el análisis los datos socio-demográficos y otras variables disponibles en las bases de datos que fueran identificadas como factores de riesgo conocidos para la infección por leptospirosis, con el fin de describir el comportamiento de los casos positivos frente a estas variables. Por otro lado, se realizaron entrevistas con expertos para ver la posibilidad de explorar variables alternativas no disponibles en las bases de datos, pero cuyo dato ecológico estuviese disponible en otros estudios.

\section{Resultados}

De todas las determinaciones para infección por leptospirosis realizadas en Valdivia (n: 2.889) se parearon 2.694 con la base de datos del Estudio de Enfermedades Prioritarias 2003 (n: 3.619) (Figura 1). La distribución por grupo etario y sexo de esta muestra fue concordante con la distribución por sexo y edad obtenida en el Estudio de Prevalencia de Enfermedades Prioritarias 2003 (Tabla 2). Del total de muestras analizadas, 6 resultaron positivas, determinando una prevalencia nacional de portadores de anticuerpos para leptospirosis de $0,4 \%$ (IC 95\%: 0,1-1,9) (Tabla 3). De los casos identificados como positivos, todos pertenecían al sexo femenino, con un rango de edad entre 26 y 86 años y eran mayoritariamente casadas. Sólo una era trabajadora dependiente y el resto se dedicaba a labores domésticas, jubiladas o están cesantes. Tres de los 6 casos identificados vivían en la Región del Libertador B. O’Higgins ( $\mathrm{VI}^{\circ}$ Región), en las comunas de Pichidegua,

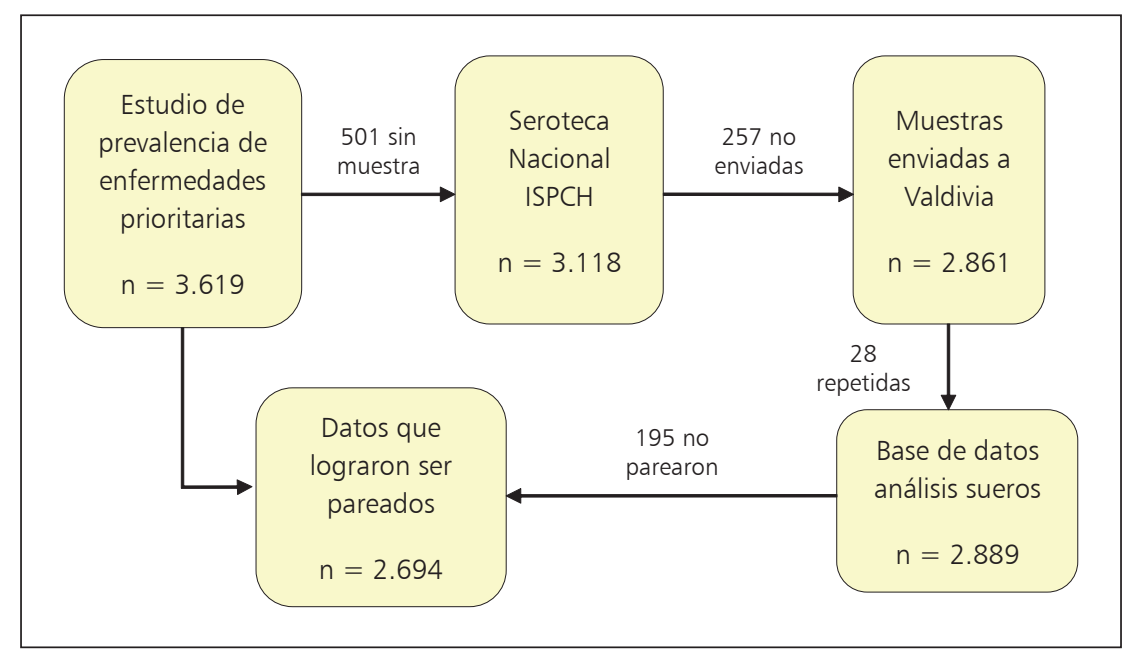

Figura 1. Algoritmo de creación de base de datos. 
Tabla 2. Distribución por edad y sexo de la muestra analizada para Leptospirosis spp. y de la Encuesta Nacional de Enfermedades Prioritarias 2003

\begin{tabular}{|llcc} 
& Grupos & \% muestra (IC 95\%) & \% Encuesta 2003 (IC 95\%) \\
Edad & $17-24$ & $19,5(16,9-22,4)$ & $18,9(17,6-20,2)$ \\
(años) & $25-44$ & $41,9(38,9-45,0)$ & $43,5(41,9-45,1)$ \\
& $45-64$ & $27,6(25,3-30,0)$ & $26,7(25,3-28,1)$ \\
& $>$ de 64 & $10,9(9,7-12,3)$ & $10,9(9,9-11,9)$ \\
\multirow{2}{*}{ Sexo } & Masculino & $48,7(46,0-51,4)$ & $45,5(43,9-47,1)$ \\
& Femenino & $51,3(48,6-54,0)$ & $54,5(52,9-56,1)$ \\
\hline
\end{tabular}

Marchihue y Navidad. Un caso detectado pertenecía a la Región Metropolitana, uno a la Región del Maule (VII ${ }^{\circ}$ Región) y uno en la Región de Araucanía (IX ${ }^{\circ}$ Región). Cuatro de 6 casos ocurrieron en áreas urbanas, de los cuales 3 fueron reactivos al serovar icterohaemorrhagiae. En 2 de 6 casos se identificó anticuerpos para el serovar pomona y en 1 de 6 el serovar bratislava.

Para 3 de los casos positivos se pudieron obtener antecedentes socioeconómicos a partir de la Encuesta de Calidad de Vida y Salud realizada el año 2000 (Tabla 4). Los casos identificados pertenecían al nivel socioeconómico (NSE) medio y bajo. Dos de los casos refirieron tener preocupaciones por los perros vagos, ratones y moscas y por los malos olores en ese entonces. Los casos de los cuales no se obtuvo información de la Encuesta de Calidad de Vida y Salud (caso 1, caso 2 y caso 3 ) se pudo obtener el NSE del hogar, a partir del estudio de Prevalencia de Enfermedades Prioritarias 2003, siendo los tres casos de nivel D (medio-bajo).

No se encontraron casos en la zona norte del país, siendo todos los casos positivos de comunas de la zona central o sur. Los 3 casos que ocurrieron en la Región del Libertador B. O’Higgins, provenían de comunas con un alto porcentaje de ruralidad. Los casos que ocurrieron en las regiones de Bío Bío $\left(\mathrm{VIII}^{\circ}\right)$ y Araucanía $\left(\mathrm{IX}^{\circ}\right.$ ) provenían de comunas donde el porcentaje de población total de pobres y de hogares pobres es igual o mayor que el promedio del país (Tabla 5).

\section{Discusión}

Este es el primer reporte de prevalencia nacional de leptospirosis. En este estudio de población general en base al reconocimiento de IgG anti leptospira se encontró una baja prevalencia nacional de portación de anticuerpos. Debido al pequeño número de muestras positivas y la utilización de los factores de expansión para el cálculo, el margen de error de la prevalencia estimada es amplio (IC 95\%: 0,1-1,9\%), pero aún así, las cifras continúan siendo bajas. Este diagnóstico epidemiológico difiere de otros reportados en países con climas tropicales, donde hay mayor endemia y alto riesgo de brotes estacionales ${ }^{1-3}$. El serovar que se encontró con mayor frecuencia fue icterohaemorrhagiae, lo cual se condice con lo que sucede en los casos notificados por ENO para el año $2006^{24}$.

En este análisis, todos los casos positivos para el serovar icterohaemorrhagiae provenían de áreas urbanas, donde la densidad de habitantes es mayor. Asociado a esto, se ha demostrado que la población de ratones que convive con poblaciones humanas es la especie Rattus rattus, quienes, a su vez, son los principales portadores de este serovar. Por otro lado, se ha observado que los caninos también son un reservorio importante de este serovar, pudiendo ser una fuente de infección, principalmente en casos no ocupacionales ${ }^{28,29}$.

Vale la pena destacar que dos casos resultaron positivos para el serovar bratislava, un serovar poco frecuente y asociado a porcinos, equinos y caninos ${ }^{30-33}$. Este serovar fue el segundo en frecuencia en estos casos, a diferencia de los casos notificados, donde el segundo lugar lo ocupó el serovar canicola $^{24}$.

$\mathrm{Al}$ analizar los factores de riesgo que han sido descritos en la literatura médica y compararlos con los datos obtenidos en este estudio, se observa que se describe un patrón diferente a lo que comúnmente se reporta en la literatura científica, ya que los afectados eran todas mujeres y que se desempeñaban en trabajos domésticos, estaban jubiladas o cesantes. Aunque esto no signifique que estas mujeres no realizaran ningún tipo de trabajo donde exis-

\begin{tabular}{|c|c|c|c|c|c|c|c|}
\hline Caso (Folio) & $\begin{array}{c}\text { Edad } \\
\text { (años) }\end{array}$ & Sexo & $\begin{array}{l}\text { Estado } \\
\text { civil }\end{array}$ & Actividad & Comuna - Región - Zona & Nivel educacional & Título y Serovar \\
\hline $1(7713)$ & 46 & Femenino & Casada & Dueña de casa & Pichidegua - VI - Rural & $4^{\circ}$ Básico & 1:50 pomona \\
\hline $2(7768)$ & 58 & Femenino & Casada & Trabajadora dependiente & Marchihue - VI - Urbana & $4^{\circ}$ Humanidades & 1:50 icterohaemorrhagiae \\
\hline $3(8317)$ & 26 & Femenino & Soltera & Cesante & Angol - IX - Urbana & $4^{\circ}$ Medio Científico Humanista & 1:50 icterohaemorrhagiae \\
\hline $4(2448)$ & 69 & Femenino & Casada & Dueña de casa & Navidad - VI - Rural & $8^{\circ}$ Básico & 1:50 bratislava \\
\hline $5(2913)$ & 86 & Femenino & Viuda & Jubilada & Chillán - VIII - Urbana & Analfabeto, sin estudios & $1: 50$ bratislava \\
\hline $6(5354)$ & 35 & Femenino & Casada & Cesante & Providencia - RM - Urbana & $4^{\circ}$ Medio Técnico Profesional & 1:100 icterohaemorrhagiae \\
\hline
\end{tabular}




\begin{tabular}{|c|c|c|c|}
\hline Variable & Caso 4 & Caso 5 & Caso 6 \\
\hline NSE & $\mathrm{D}$ & $\mathrm{CB}$ & B \\
\hline NSE_INE & Bajo & Medio & Medio \\
\hline Tipo de vivienda - material & $\begin{array}{l}\text { Casa de ladrillo, concreto, } \\
\text { bloque o piedra }\end{array}$ & Casa de ladrillo, concreto, bloque o piedra & $\begin{array}{l}\text { Departamento de ladrillo, concreto, } \\
\text { bloque o piedra }\end{array}$ \\
\hline Calificación de las viviendas en la manzana & Rural & Modestas & Medio alta \\
\hline Estado de las viviendas de las manzanas & Rural & Regulares & Buenas \\
\hline Calificación de las veredas & Rural & Regulares & Buenas \\
\hline Preocupaciones & No manifiesta & $\begin{array}{l}\text { Presencia de perros vagos, moscas, ratones, etc.; } \\
\text { limpieza de calles y veredas; malos olores }\end{array}$ & $\begin{array}{l}\text { Presencia de perros vagos, moscas, } \\
\text { ratones, etc.; malos olores }\end{array}$ \\
\hline
\end{tabular}

tiera riesgo de contagio de leptospirosis, ninguna de ellas reportó realizar actividades de riesgo para la transmisión de esta enfermedad como lo son actividades agrícolas, veterinarios, limpiadores de alcantarillados, trabajadores de mataderos y des-ratizadores ${ }^{8}$. Esto puede explicarse debido a que en esta muestra lo que se midió fue la portación de anticuerpos, la cual puede permanecer por años luego de la infección, y no el diagnóstico de la enfermedad la cual se da predominantemente en el sexo masculino ${ }^{27}$.

En esta serie de casos, la mayoría de los afectados pertenecía a un nivel socioeconómico medio-bajo y bajo y algunos relataron una preocupación por perros vagos, ratones y malos olores cerca de su lugar de residencia. Aunque no se puede predecir que estos factores sean la causa de la infección de estos pacientes, estas características han sido descritas como factores de riesgo para la enfermedad $^{34,35}$. También se describió que los individuos, en su mayoría, residían en comunas que combinan ruralidad y pobreza, lo cual podría corresponder a un grupo de mayor riesgo para adquirir leptospirosis en Chile.

Cuando se evalúan los títulos de anticuerpos presentes en estos casos, se observa que todos los casos presentaron títulos bajos de anticuerpos, lo cual podría desechar la teoría de infecciones sintomáticas agudas donde, generalmente, los títulos de anticuerpos alcanzan niveles más elevados $^{27}$. Los niveles de 1:100 y 1:50 observados en estos casos podrían corresponder a cuadros subclínicos de la enfermedad o, más aún, a individuos portadores de anticuerpos con antecedentes de un cuadro previo de infección por leptospiras. Se ha demostrado que los humanos pueden ser portadores de los anticuerpos por varios años luego de haber padecido la enfermedad ${ }^{1,13,27}$. Esto hace difícil predecir factores de riesgo en esta muestra, ya que no se cuenta con datos primarios recolectados para este fin. Sólo permite comparar los datos descritos en esta serie de casos con los factores de riesgo reportados en la literatura científica.

\begin{tabular}{|c|c|c|c|c|c|}
\hline \multirow[t]{2}{*}{ Región } & \multirow[t]{2}{*}{ Comuna } & \multirow[t]{2}{*}{ Zona } & \multirow{2}{*}{$\begin{array}{l}\text { Ruralidad } \\
\text { (\%) }\end{array}$} & \multicolumn{2}{|c|}{ Pobreza } \\
\hline & & & & $\begin{array}{c}\text { Población total } \\
\text { (\%) }\end{array}$ & $\begin{array}{l}\text { Hogares } \\
(\%)\end{array}$ \\
\hline RM & Providencia & Urbana & 0 & 3,5 & 2 \\
\hline VI & Navidad & Rural & 87,03 & 16,8 & 13,4 \\
\hline $\mathrm{VI}$ & Marchihue & Urbana & 68,02 & 10,6 & 8,2 \\
\hline $\mathrm{VI}$ & Pichidegua & Rural & 72,04 & 17,5 & 14,2 \\
\hline VIII & Chillán & Urbana & 8,61 & 18,7 & 15,7 \\
\hline IX & Angol & Urbana & 10,6 & 22,7 & 19,7 \\
\hline Total país & & & 13 & 18,7 & 15,3 \\
\hline
\end{tabular}

La detección de anticuerpos a través de la técnica MAT es considerada la técnica de elección para este procedimiento. Sin embargo, existen algunas limitaciones propias de este método que podrían interferir en los resultados: uno de los principales problemas es la probabilidad de un resultado falsamente negativo debido a que no se incluyó en el panel el o los serovares adecuados ${ }^{1}$. En este estudio se incluyeron los serovares más comúnmente descritos en Chile (hardjo, pomona, canicola, ballum, icterohaemorrhagiae, autumnalis, grippotyphosa, tarassovi y bratislava). Sin embargo, esto no excluye la posibilidad de que existan nuevos serovares que estén causando esta enfermedad. Por otro lado, la posibilidad de falsos negativos o de reacciones cruzadas es de mayor importancia en el caso de infecciones sintomáticas agudas, donde se requiere un diagnóstico temprano de la enfermedad y la seroconversión puede demorar hasta 7 días ${ }^{1,19,21}$. En este caso, los bajos títulos obtenidos se inclinan más hacia una portación crónica de anticuerpos, donde la seroconversión ya ha sucedido. Los casos de infecciones agudas donde la seroconversión no ha ocurrido, o de infecciones pasadas donde los títulos de anticuerpos ya han disminuido a niveles muy bajos, 
podrían no haber sido detectados y, por lo tanto, no fueron considerados en el cálculo de la prevalencia.

\section{Conclusión}

Este es el primer trabajo de prevalencia nacional de anticuerpos contra leptospirosis realizado en Chile. La prevalencia nacional de leptospirosis en población general de acuerdo a las técnicas utilizadas es baja. El serovar más frecuente fue el serovar icterohaemorrhagiae, seguido de los serovares bratislava y pomona.

Este análisis nos da un diagnóstico de situación de lo que está ocurriendo en nuestro país. Sin embargo, no permite concluir sobre factores de riesgo asociados a la infección, sólo permite describir algunas características presentes en la muestra que se corresponden con factores de riesgo para la infección. Asimismo, sería conveniente realizar estudios focalizados para indagar factores de riesgo para leptospirosis, como por ejemplo, la presencia de animales domésticos, actividades recreacionales o laborales y en zonas donde exista un ambiente favorable para la sobrevivencia de Leptospira spp, a fin de poder identificar a los grupos de riesgo y llevar a cabo un programa educativo, tanto en profesionales de la salud como en la comunidad.

Agradecimientos. A Pedro Cattan y Paola Correa del Departamento de Ciencias Biológicas Animales de la Facultad
Ciencias Veterinarias y Pecuarias de la Universidad de Chile por su aporte en el análisis de los datos. A Marcela Ferrada, Magister Epidemiología, Facultad de Medicina, Pontificia Universidad Católica de Chile por su aporte en la recopilación de la información.

\section{Resumen}

Introducción: La leptospirosis es una zoonosis endémica en ciertas regiones de Chile. Desde que se incorporó esta enfermedad en la lista de las Enfermedades de Notificación Obligatoria (ENO) se ha logrado mantener una adecuada vigilancia. Sin embargo, existen casos subclínicos y con manifestaciones inespecíficas que no son reportados. Objetivo: Determinar la prevalencia nacional de leptospirosis y conocer características epidemiológicas de individuos seropositivos. Material y Métodos: Análisis de datos secundarios obtenidos en la Encuesta Nacional de Enfermedades Prioritarias, 2003. Resultados: La prevalencia nacional de leptospirosis fue $0,4 \%$. Las características que se identificaron con mayor frecuencia en el grupo de personas positivas fueron nivel socio-económico bajo y sexo femenino. Los serovares más frecuentes fueron icterohaemorrhagiae, bratislava y pomona. Conclusión: Este es el primer trabajo de prevalencia nacional de leptospirosis realizado en Chile. Este estudio aporta al diagnóstico de situación de esta patología en nuestro país.

\section{Referencias bibliográficas}

1.- World Health Organization and International Leptospirosis Society. Human leptospirosis: guidance for diagnosis, surveillance and control 2003; http://whqlibdoc.who.int/hq/2003/WHO CDS_CSR_EPH_2002.23.pdf.

2.- Platts-Mills J A, LaRochelle P, Campos K, Vinetz J M, Gotuzzo E, Ricaldi J N. Seroprevalence of leptospirosis in Puente Piedra, Lima, in 2006. Rev Peru Med Exp Salud Pública 2011; 28 (2): 273-6.

3.- Romero M H, Sánchez J A, Hayek L C. The prevalence of antibodies against Leptospira in urban human and canine populations from the Tolima Department. Rev Salud Publica (Bogotá) 2010; 12 (2): 268-75.

4.- Morshed M G, Konishi H, Terada Y, Arimitsu Y, Nakazawa T. Seroprevalence of leptospirosis in a rural flood prone district of Bangladesh. Epidemiol Infect 199; 112 (3): 527-31.

5.- Navarrete-Espinosa J, Acevedo-Vales J A, Huerta-Hernández E, Torres-Barranca J, Gavaldón-Rosas DG. Prevalence of dengue and leptospira antibodies in the state of Veracruz,
México. Salud Pública Mex 2006; 48 (3): 220-8.

6.- Cacciapuoti B, Ciceroni L, Pinto A, Apollini M, Rondinella V, Bonomi U, et al. Survey on the prevalence of leptospira infections in the Italian population. Eur J Epidemiol 1994; 10 (2): 173-80.

7.- Kawaguchi L, Sengkeopraseuth B, Tsuyuoka R, Koizumi N, Akashi H, Vongphrachanh P, et al. Seroprevalence of leptospirosis and risk factor analysis in flood-prone rural areas in Lao PDR. Am J Trop Med Hyg 2008; 78 (6): 957-61.

8.- Perret C, Abarca K, Dabanch J, Solari V, García P, Carrasco S, et al. Prevalencia y presencia de factores de riesgo de leptospirosis en una población de riesgo de la Región Metropolitana. Rev Med Chile 2005; 133 : 426-31.

9.- Thai K T, Binh T Q, Giao P T, Phuong H L, Hung le Q, Van Nam N, et al. Seroepidemiology of leptospirosis in southern Vietnamese children. Trop Med Int Health 2006; 11 (5): 738-45.

10.- Swapna R N, Tuteja U, Nair L, Sudarsana J. Seroprevalence of leptospirosis in high risk groups in Calicut, North Kerala, India. Indian J Med Microbiol 2006; 24: 349-52.
11.- Lévesque B, Messier V, Bonnier-Viger Y, Couillard M, Côté S, Ward B J, et al. Seroprevalence of zoonoses in a Cree community (Canada). Diagn Microbiol Infect Dis 2007; 59 (3): 283-6.

12.- Gonçalves D D, Teles P S, dos Reis C R, Lopes F M, Freire R L, Navarro I T, et al. Seroepidemiology and occupational and environmental variables for leptospirosis, brucellosis and toxoplasmosis in slaughterhouse workers in the Paraná State, Brazil. Rev Inst Med Trop Sao Paulo 2006; 48 (3): 135-40.

13.- Riedemann S, Zamora J. Aspectos epidemiológicos de leptospirosis humana en el medio rural. Zbl Vet Med B 1982; 29: 702-7.

14.- Zamora J, Riedemann S, Montecinos M I, Cabezas X. Encuesta serológica de leptospirosis humana en ocupaciones de alto riesgo en Chile. Rev Med Chile 1990; 118: 247-52.

15.- Zamora J, Riedemann S, Leal H, Jackson C. Evidencia serológica de leptospirosis en labradores de arrozales. Rev Med Chile 1988; 116: 723-8.

16.- Silva H R, Tavares-Neto J, Bina J C, Meyer R. Leptospiral infection and subclinical 
presentation among children in Salvador, Bahia. Rev Soc Bras Med Trop 2003; 36 (2): 227-33.

17.- World Health Organization. Leptospirosis worldwide, 1999. WER 1999; 74: 237-42.

18.- de Souza A I, Nogueira J M, Pereira M M. Anti-Leptospira antibodies in patients in the State of Mato Grosso do Sul with clinical suspicion of dengue or viral hepatitis. Rev Soc Bras Med Trop 2007; 40 (4): 431-5.

19.- Zunino E, Pizarro R. Leptospirosis: Puesta al día. Rev Chilena Infectol 2007; 24 (2): 220-6.

20.- Riedemann S, Zamora J. Leptospirosis animal. Serogrupos y serovares presentes en Chile y su importancia. Arch Med Vet 1987; 19: 69-72.

21.- Bharti A R, Nally J E, Ricaldi J N, Matthias M A, Díaz M M, Lovett M A, et al. Leptospirosis: a zoonotic disease of global importance. Lancet Infect Dis 2003; 3 (12): 757-71.

22.- Arias H, Núñez M, Valenzuela I, Olivares A. Brote epidémico de leptospirosis en niños de Linares. Rev Chil Pediatr 2003; 74 (4): 405-10.

23.- Navarrete G. Vigilancia de leptospirosis. Departamento de Epidemiología. MINSAL. 2002;4F/03.
24.- Olea A. Zoonosis y Enfermedades de Transmisión Vectorial. El Vigía 2007; 25 (10): 52-6.

25.- Departamento de Epidemiología. Ministerio de Salud. Encuesta Nacional de Salud, Chile, 2003. 2004; Available at: http://epi.minsal.cl/epi/html/ invest/ENS/ENS.htm. (Accedido agosto de 2012).

26.- Departamento de Epidemiología. Ministerio de Salud. Encuesta Calidad de Vida, Chile, 2001. 2002; Available at: http://epi.minsal.cl/epi/html/ sdesalud/cdevid/encuescdv.htm. /Accedido agosto de 2012).

27.- Levett P N. Leptospirosis. Clin Microbiol Rev 2001; 14 (2): 296-326.

28.- Acha P, Szyfres B. Zoonoses and Communicable Diseases Common to Man and Animals. Third edition ed. Washington, DC: Organización Panamericana de la Salud; 2001: p. $175-85$

29.- Silva R F, Riedemann S. Seroprevalencia de leptospirosis canina en perros atendidos en clínicas veterinarias, mediante aglutinación microscópica y comparación con las técnicas de aislamiento e inmunofluorescencia indirecta. Arch Med Vet 2007; 39 (3): 269-74.
30.- Stokes J E, Kaneene J B, Schall W D, Kruger J M, Miller R, Kaiser L, et al. Prevalence of serum antibodies against six Leptospira serovars in healthy dogs. J Am Vet Med Assoc 2007; 230 (11): 1657-64.

31.- Cisneros Puebla M A, Moles Cervantes L P, Rosas D G, Serranía N R, Torres-Barranca J. Diagnostic serology of swine leptospirosis in Mexico 1995-2000. Rev Cubana Med Trop 2002; 54 (1): 28-31.

32.- Goldstein R E, Lin R C, Langston C E, Scrivani P V, Erb H N, Barr S C. Influence of infecting serogroup on clinical features of leptospirosis in dogs. J Vet Intern Med 2006; 20 (3): 489-94.

33.- Odontsetseg N, Boldbaatar D, Mweene A S, Kida H. Serological prevalence of Leptospira interrogans serovar Bratislava in horses in Mongolia. Vet Rec 2005; 157 (17): 518-9.

34.- Plank R, Dean D. Overview of the epidemiology, microbiology, and pathogenesis of Leptospira spp. in humans. Microbes Infect 2000; 2 (10): 1265-76.

35.- Sehgal S C. Epidemiological patterns of leptospirosis. Indian J Med Microbiol 2006; 24 (4): 310-11. 